\title{
Wavelet-based multiscale similarity measure for complex networks
}

\author{
Ankit Agarwal ${ }^{1,2,3, a}$, Rathinasamy Maheswaran ${ }^{1,4}$, Norbert Marwan ${ }^{1}$, Levke Caesar ${ }^{1,5}$, \\ and Jürgen Kurths ${ }^{1,2,6}$ \\ ${ }^{1}$ Potsdam Institute for Climate Impact Research (PIK), Member of the Leibniz Association, Telegrafenberg, \\ Potsdam, Germany \\ ${ }^{2}$ Institute of Earth and Environmental Science, University of Potsdam, Potsdam, Germany \\ ${ }^{3}$ GFZ German Research Centre for Geosciences, Section 5.4: Hydrology, Telegrafenberg, Potsdam, Germany \\ ${ }_{5}^{4}$ Civil Engineering Department, MVGR College of Engineering, Vizianagaram, India \\ ${ }^{5}$ Institute of Physics and Astronomy, University of Potsdam, Potsdam, Germany \\ ${ }^{6}$ Institute of Physics, Humboldt Universität zu Berlin, Berlin, Germany
}

Received 16 July 2018 / Received in final form 6 September 2018

Published online 26 November 2018

(C) EDP Sciences / Società Italiana di Fisica / Springer-Verlag GmbH Germany, part of Springer Nature, 2018

\begin{abstract}
In recent years, complex network analysis facilitated the identification of universal and unexpected patterns in complex climate systems. However, the analysis and representation of a multiscale complex relationship that exists in the global climate system are limited. A logical first step in addressing this issue is to construct multiple networks over different timescales. Therefore, we propose to apply the wavelet multiscale correlation (WMC) similarity measure, which is a combination of two state-of-the-art methods, viz. wavelet and Pearson's correlation, for investigating multiscale processes through complex networks. Firstly we decompose the data over different timescales using the wavelet approach and subsequently construct a corresponding network by Pearson's correlation. The proposed approach is illustrated and tested on two synthetics and one real-world example. The first synthetic case study shows the efficacy of the proposed approach to unravel scale-specific connections, which are often undiscovered at a single scale. The second synthetic case study illustrates that by dividing and constructing a separate network for each time window we can detect significant changes in the signal structure. The real-world example investigates the behavior of the global sea surface temperature (SST) network at different timescales. Intriguingly, we notice that spatial dependent structure in SST evolves temporally. Overall, the proposed measure has an immense potential to provide essential insights on understanding and extending complex multivariate process studies at multiple scales.
\end{abstract}

\section{Introduction}

Networks provide analytical capabilities to uncover structure and patterns of geophysical processes in which nodes denote geographic locations, and links indicate connections between these locations not by spatial proximity but rather on the basis of a similar dynamics in the process. The strength of the connection (link) is estimated using similarity measures that quantify the interrelation between the two nodes. In the past, several similarity measures have been investigated in the context of climate networks. Most prominent include Pearson's correlation coefficient (PCC) [1-6] mean distance [7] event synchronization [8-10] spike synchronization [11], mutual information $[1,12-15]$ and more recently multiscale event synchronization [16]. The selection of the similarity measure is generally a function of (a) characteristics of the data (Gaussian, non-Gaussian) and (b) nature of relationships

\footnotetext{
${ }^{a}$ e-mail: agarwal@pik-potsdam.de
}

(linear, nonlinear, or multiscale, etc.) between the nodes in the networks [17]. For example, nonlinear connections between nodes are better captured by mutual information than the linear counterpart correlation. Malik et al. showed that for signals having an event like structure (non-Gaussian data) such as extreme precipitation, event synchronization performs better than PCC, as it statistically measures the coincidence of single events and uses a dynamic (not fixed) time delay $[9,18]$. Most of the methods mentioned above have been successfully applied in the construction of networks in order to understand several complex processes [3,9,19-23]. However, the present set of network node similarity measures are limited to capture multiscale relationships in Gaussian data that are prominent in many natural processes. In the past, several studies have reported that complex systems, in general, are governed by coupling mechanisms, and these couplings are typically exhibited at unique temporal and spatial scales $[5,24,25]$. For example, Molini et al. investigated the presence of causality across rainfall timescales and 
showed a significant influence of coarser time scales on the finer scaled rainfall variability [26]. Similarly, Varotsos et al. showed the presence of information transfer from larger to smaller time scales in the global daily mean surface air temperature [27]. Hatla et al. and Sturtevant et al. identified the presence of scale-emergent processes in wetland methane exchange processes $[28,29]$. On similar lines, a study [30] revealed the dynamics of the soil moisture-temperature coupling over a wide range of temporal scales (from days to several months). More recently, Paluš showed evidence of scale-specific relationship emerging between sea surface temperature (SST) and large-scale climate oscillations such as El Niño/Southern Oscillation (ENSO) and North Atlantic Oscillation (NAO) [14]. These studies strengthen the hypothesis of the presence of multiscale processes in geophysical systems. Casagrande et al., Miralles et al., Okin et al., and Peters et al. argue that multiscale processes can act as a triggering mechanism for extreme events, abrupt regime transition, and pattern formation [30-33]. Therefore, it is essential to investigate the process at several dominant temporal scales for improved understanding and modeling.

Advancing towards these goals will necessitate the development of a more detailed measure, which can unravel underlying multiscale relationships and changes in network structure over time. This is achieved using wavelets that obtain a set of scales reflecting the underlying oscillations at different scale levels [34-36]. In the past, wavelets based multiscale correlation and coherence have already been successfully used to characterize the temporal scales interaction between extreme events and large-scale climatic oscillations [14,26,37-46].

In this study, we suggest applying a multiscale similarity measure using wavelets and PCC for network construction. In wavelet multiscale correlation (WMC) measure, wavelets are used to isolate the time scales of variation and correlation to identify the node-node interactions at these scales. This would help in systematically (1) identifying the dominant processes and the timescales they act on, and (2) understanding how the network evolves with temporal scales. The multiscale similarity measure is applied to two prototypical examples and a real-time case study of SST networks. The results are finally compared with the results derived with simple Pearson correlation.

The essential background on wavelet is discussed in Section 2; Section 3 is devoted to testing the performance of the proposed approach in assessing scale-by-scale interaction in a synthetic network of known multiscale coupling. Following the test on synthetic data, Section 4 deals with the multiscale nature of SST. Finally, a further discussion of wavelet cross-correlation strength and criticality is provided in Section 5, together with concluding remarks and an outlook on future developments.

\section{Methodology}

\subsection{Wavelet transform}

In order to develop WMC as a similarity measure for network formation in analyzing complex systems, the methods of wavelet analysis [38] and PCC are combined. This section provides an introduction to the basic concepts of the wavelet analysis relevant to this study. We use the maximal overlapping discrete wavelet transform (MODWT) which is a modification of the discrete wavelet transform (DWT) [44,47]. We prefer MODWT to DWT because pyramidal structures of coefficients limit DWT. More clearly, in DWT, decimation is carried out so that only half of the coefficients of the detailed component are left at the current level, and half of the coefficients of the smooth version are recursively processed using high pass and low pass filters for coarser resolution levels. Due to this decimation, the number of wavelet coefficients is halved with each move to a coarser level [48]. This problem may be overcome by using the stationary maximal overlap discrete wavelet transform (MODWT) where the resulting gaps are filled using redundant information obtained from the original series.

MODWT decomposes the time series into different time scales or frequency components. The wavelet decomposition is realized using the two basis functions known as father wavelet $(\varphi(t))$ and mother wavelet $(\psi(t))$. The general admissibility conditions for $\psi$ to be called a wavelet function are

$$
\begin{gathered}
\int_{-\infty}^{\infty} \psi(t) d t=0 . \\
\int_{-\infty}^{\infty}|\psi(t)|^{2} d t=1 .
\end{gathered}
$$

Any function $f(t)$ can be expressed through these basis functions and their scaled and translated versions are

$$
\begin{aligned}
f(t)= & \sum_{k} s_{J, k} \varphi_{J, k}(t)+\sum_{k} d_{J, k} \psi_{J, k}(t) \\
& +\sum_{k} d_{J-1, k} \psi_{J-1, k}(t) \cdots+\sum_{k} d_{1, k} \psi_{1, k}(t)
\end{aligned}
$$

where $J$ is the total number of scales to be analyzed, and $k$ is in the range of 1 to $l$ (length of the time series). The coefficients $s_{J, k}$ are the approximation coefficients and $d_{J, k}, \ldots, d_{1, k}$ are the wavelet transform coefficients at scales $J$ to 1 , while the functions $\varphi_{J, k}(t)$ and $\psi_{j, k}(t) \mid$ $j=1, \ldots, J-1, J\}$ are the basis functions which are obtained through translation and dilation of the father $(\varphi(t))$ and mother $(\psi(t))$ wavelet function, respectively.

The mother wavelet is scaled (or dilated) by a factor of $j$ and translated (or shifted) by a factor of $k$ to give

$$
\psi_{j, k}(t)=2^{-j / 2} \psi\left(2^{-j} t-k\right) .
$$

Further, the values of the wavelet transform coefficients at each of the scales and the approximation coefficients at scale $J$ are estimated by

$$
d_{j, k} \approx \int \psi_{j, k}(t) f(t) d t, j=1, \ldots, J-1, J
$$




$$
s_{J, k} \approx \int \varphi_{J, k}(t) f(t) d t
$$

where the scaling coefficients $s_{J, k}$, which are also called smooth coefficients, capture the smooth trend of the time series at the coarse scale $2^{J}$; and the wavelet coefficients $d_{j, k}$, also known as detail coefficients, can detect deviations from the coarsest scale to the finest one.

The original series $f(t)$ can be reconstructed by summing up the detailed components and the smooth components:

$$
f(t)=S_{J, k}+D_{J, k}+D_{J-1, k}+\cdots+D_{1, k},
$$

where $S_{J, k}=\sum_{k} s_{J, k} \varphi_{J, k}(t), \quad D_{J, k}=\sum_{k} d_{J, k} \psi_{J, k}(t)$, $\ldots, D_{1, k}=\sum_{k} d_{1, k} \psi_{1, k}(t)$.

\subsection{Wavelet multiscale correlation}

Consider two time series $\{X(t)\}$ and $\{Y(t)\}$ with the same length $T$. The WMC measure between both time series can be estimated as $[34,35]$

$$
\mathrm{WMC}=\rho_{X, Y}^{l_{j}} \equiv \frac{\operatorname{COV}_{X, Y}^{l_{j}}}{\operatorname{Var}_{Y}^{l_{j}} \operatorname{Var}_{X}^{l_{j}}},
$$

where

$$
\begin{gathered}
\operatorname{Var}_{X}^{l_{j}} \equiv \frac{1}{\grave{T}_{j}} \sum_{t=M_{j}-1}^{T-1}\left[d_{j, t}^{X}\right]^{2}, \\
\operatorname{Var}_{Y}^{l_{j}} \equiv \frac{1}{\grave{T}_{j}} \sum_{t=M_{j}-1}^{T-1}\left[d_{j, t}^{Y}\right]^{2}, \\
\operatorname{COV}_{X, Y}^{l_{j}} \equiv \frac{1}{\grave{T}_{j}} \sum_{t=M_{j}-1}^{T-1} d_{j, t}^{X} d_{j, t}^{Y} .
\end{gathered}
$$

$d_{j, t}^{(.)}$denotes the MODWT wavelet coefficient of variables $\{X, Y\}$ at scale $l_{j} ; \dot{T}_{j}=T-M_{j}+1$ stands for the number of coefficients unaffected by the boundary; $M_{j}=$ $\left(2^{j}-1\right)(M-1)+1$ represents the length of the scale $l_{j}$ wavelet filter, and $M$ is the width of the wavelet filter. The multiscale correlation measure $\rho_{X, Y}^{l_{j}}$ denotes the scale-wise correlation between $X$ and $Y$ at different $l_{j}$ scales. Like the PCC, the value of $\rho_{X, Y}^{l_{j}}$ ranges between -1 and 1 . $\rho_{X, Y}^{l_{j}}=0$ implies the variables $X$ and $Y$ are not correlated at scale $l_{j} \cdot \rho_{X, Y}^{l_{j}}=1$ and $\rho_{X, Y}^{l_{j}}=-1$ indicate that the variables are perfectly correlated and anti-correlated, respectively. The values of $\left\{\rho_{X, Y}^{1 l_{j}} \ldots \rho_{X, Y}^{l_{j}}\right\}$ indicate the strength of the relation between $X$ and $Y$ at different temporal scale bands.

\subsection{Network construction}

In the context of the application of WMC as a network measure, consider a network $Z=\{N, E\}$ containing a set of $N$ nodes together with a set of $E$ edges $\{j, k\}$ which are 2 element subsets of $N$. Let each of the nodes be characterized by a time series of a certain variable of interest. The WMC between two nodes is estimated using equation (8).

We begin the network construction at each temporal scale by finding the WMC measure between each pair of nodes. Since an inverse relationship is equally relevant in the present application, we set the edge weight to absolute values of WMC $\left|\rho_{X, Y}^{l_{j}}\right|[6,49]$. By doing so, we generate the $N \times N$ matrix of $\rho^{l_{j}}$ values for each scale $l j$. We are only interested in the highly correlated links. Therefore, we apply a threshold to the $\left|\rho^{l_{j}}\right|$ values which is the $\theta=95$ th percentile of all $\left|\rho^{l_{j}}\right|$ values. This converts the $\left|\rho^{l_{j}}\right|$ matrix to a binary matrix, the adjacency matrix $(A)$ of an undirected and unweighted network:

$$
A_{m, n}= \begin{cases}1, & \text { if }\left|\rho_{m, n}^{l_{j}}\right| \geq \theta^{l_{j}} \\ 0, & \text { else. }\end{cases}
$$

Here, $\theta^{l_{j}}$ is the chosen threshold for each scale $l j$, and $A_{m, n}=1$ denotes a link between the $m$ th and $n$th node and 0 denotes otherwise.

The application of the WMC as the network measure reveals the network topology at each of the $l_{j}$ scales and allows the investigation of the network at different temporal scales. In the following, we demonstrate the potential of this proposed method with prototypical examples and real world application with a finite number of nodes.

\section{Testing WMC with synthetic data}

The prototypical examples aim to test the efficacy of the proposed WMC method over the traditional Pearson's correlation as an effective network reconstruction measure while dealing with multiscale processes. Following the approach by Agarwal et al. [16], we test WMC using a set of case studies including stationary and non-stationary synthetic data [16]. The different scales are included as periodic signals of different frequency. Correlated noise is added to mimic real-world processes where stochasticity and autocorrelation are important features. The mathematical details of the case studies and wavelet power spectra are given in Table 1 and Figure 1.

Case I. We construct a synthetic network (Fig. 2) of 30 nodes $\left(X_{i}(t) ; i=1,2, \ldots, 30\right)$. For each of the nodes $\left(X_{i}(t) ; i=1,2, \ldots, 30\right)$ different time series are generated by combining a 2 nd order autoregressive process (AR) and periodic signals (Tab. 1). The mathematical expression for the periodic signal and time series formulation are provided in the Table 1.

The mathematical construction of the time series (Tab. 1) shows that 30 nodes of the network are classified into three group such that each group encompasses a particular cosine function (frequency). Group I: 
Table 1. Details of synthetic models. Here, $f$ denotes a function.

\begin{tabular}{llll}
\hline Mathematical expression & & Other details \\
\hline Periodic signals & $S 1=\cos \left(2 \pi \omega_{1}\right) ;$ & $\omega_{1}=0.4 t ;$ & Refer Figure 1 \\
& $S 2=\cos \left(2 \pi \omega_{2}\right) ;$ & $\omega_{2}=0.09 t ;$ & \\
Case I & $S 3=\cos \left(2 \pi \omega_{3}\right)$ & $\omega_{3}=0.01 t$ & \\
$(t=1: 1000)$ & $X_{i}(t)=\varphi_{1} X(t-1)+\varphi_{2} X(t-2)+\varepsilon_{t}+Y_{1}, 1<i<10$ & $Y_{1}=f(S 1) ;$ \\
& $X_{i}(t)=\varphi_{1} X(t-1)+\varphi_{2} X(t-2)+\varepsilon_{t}+Y_{2}, 11<i<20$ & $Y_{2}=f(S 2) ;$ \\
& $X_{i}(t)=\varphi_{1} X(t-1)+\varphi_{2} X(t-2)+\varepsilon_{t}+Y_{3}, 21<i<30$ & $=f(S 1, S 2) ;$ \\
Case II & $X_{i}(t)=\varphi_{1} X(t-1)+\varphi_{2} X(t-2)+\varepsilon_{t}+$ & $1<i<10$ & $Y_{1}=f(S 1) ; Y_{2}=f(S 2) ;$ \\
$(t=1: 2000)$ & $\left(Y_{1}\right)_{t=1: 1000}+\left(Y_{2}\right)_{t=1001: 2000}$ & \\
& $X_{i}(t)=\varphi_{1} X(t-1)+\varphi_{2} X(t-2)+\varepsilon_{t}+$ & $11<i<20$ & $Y_{3}=f(S 1, S 2) ; Y_{4}=f(S 2, S 3) ;$ \\
& $\left(Y_{3}\right)_{t=1: 1000}+\left(Y_{4}\right)_{t=1001: 2000}(t-2)+\varepsilon_{t}+$ & $21<i<30$ & $Y_{5}=f(S 1, S 2, S 3)($ refer Fig. 3$)$ \\
& $X_{i}(t)=\varphi_{1} X(t-1)+\varphi_{2} X(t-S)$ &
\end{tabular}
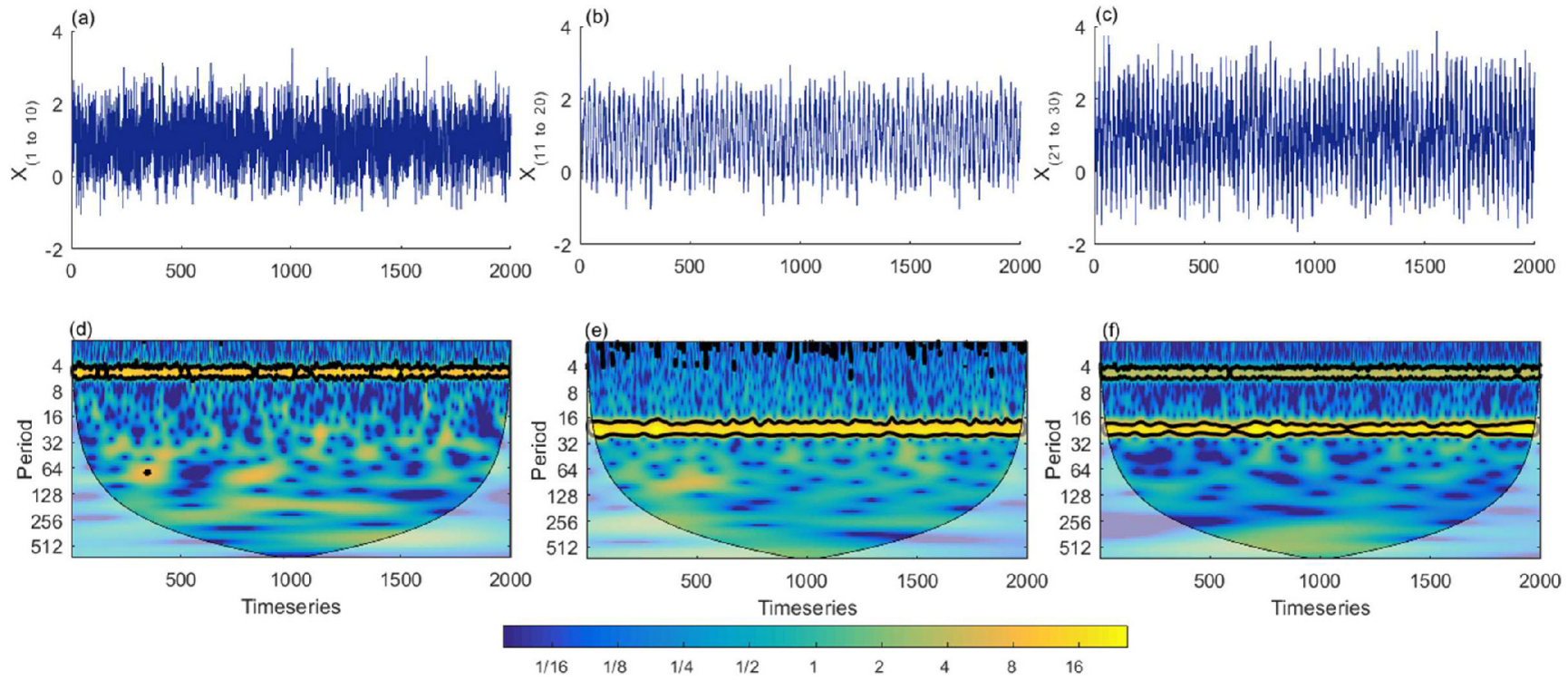

Fig. 1. The top row $(\mathrm{a}-\mathrm{c})$ shows the plot of the synthetic time series generated for network formation. Ten simulations were generated for each group of nodes. The bottom plot $(\mathrm{d}-\mathrm{f})$ shows the wavelet power spectra for one single time series from each of the group. The regions within the black contours denote the $95 \%$ significant values and the cones of influence denote the extent of the boundary distortion.

node 1 to 10, group II: node 11 to 20 , and group III: node 21 to 30 encompass the cosine functions $Y_{1}, Y_{2}$, and $Y_{3}$ with different frequencies, thus, different scales. The particular cosine functions (signal) are plotted in Figures $1 \mathrm{a}-1 \mathrm{c}$ and the corresponding power spectra are shown in Figures 1d-1f.

The $\varphi_{1}=2.5$ and $\varphi_{2}=0.4$ are autoregressive constants, while $\varepsilon_{t}$ represents white noise with the signal/noise ratio of amplitude $(\mathrm{SNR})=0.90$. The resultant node's time series have features that are often found in geophysical, hydrological, and climatic data, where auto-correlated noise and high frequency, small-scale processes are superimposed on low frequency, coarse-scale processes $[16,50]$. Such structures are widespread in time series of seismic signals, turbulence, air temperature, precipitation, hydrologic fluxes, or the ENSO. They can also be found in spatial data, e.g., in ocean waves, seafloor bathymetry, or land surface topography [50].
First, the network is constructed using absolute correlation values of PCC applying the 95th percentile threshold (Fig. 2a). Even though, group I (nodes 1 to 10, pink circles in Fig. 2) and group III (nodes 21 to 30, blue circles) nodes share a strong similarity owing to the common periodicity (signal $S 1$, Tab. 1) the network link does not reflect the expected similarity (strong connections) in the dynamics. Hence, it does not give an appropriate impression of the connection. A similar misleading result is inferred between the group II and group III nodes. Further, owing to the strong self-similarity between the nodes of group III, we observe links connecting the nodes $21-30$ with each other. However, self-similar links are absent in the group I and group II because of the low signal to noise ratio, which makes the underlying correlation.

Next, for the same 30 nodes, the similarity measure WMC, $\left|\rho_{X, Y}^{l_{j}}\right|$ (Eq. (7)), is calculated at different timescales and the corresponding network is formed using 

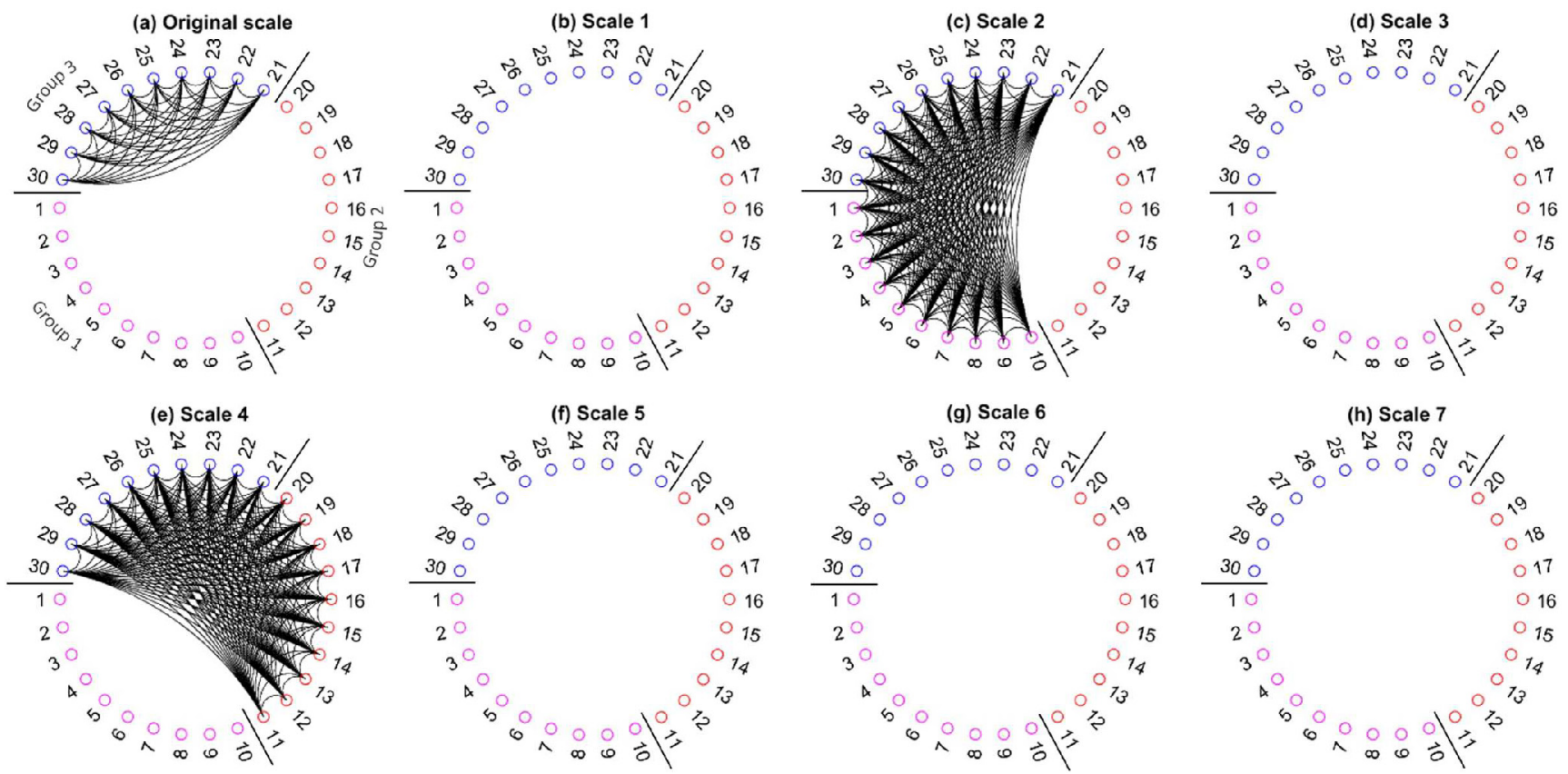

Fig. 2. Network structure and significant links obtained using the (a) PCC (original scale) and (b-h) WMC at scales $1-7$. A significant link is defined when the value of the correlation is higher than the 95th percentile.

\begin{tabular}{|c|c|c|c|}
\hline Nodes & $\mathrm{t}=1: 1000$ & $\mathrm{t}=1001: 2000$ & Signal: Scale \\
\hline 1 to 10 & S1 & $\mathrm{S} 2$ & S1: scale 2 \\
\hline 11 to 20 & S1: & S2:S3 & S2: scale 4 \\
\hline \multirow[t]{2}{*}{21 to 30} & $\begin{array}{l:ll}\mathrm{S} 1 & \mathrm{~S} 2 & \mathrm{~S} 3 \\
& \end{array}$ & $\mathrm{~S} 1 \mathrm{~S} 2 \mathrm{~S} 3$ & \multirow[t]{2}{*}{ S3: scale 7} \\
\hline & $\begin{array}{l}\text { Corres } \\
\text { tions a }\end{array}$ & $\begin{array}{l}\text { ernal connec- } \\
\text { s } 21 \text { to } 30\end{array}$ & \\
\hline
\end{tabular}

Fig. 3. Visualization of the common periodicities presents among the group of 30 nodes.

a 95 th percentile threshold (Figs. $2 \mathrm{~b}-2 \mathrm{~h}$ ). Out of 7 scales considered, the links are present only at scales 2 (period 4-8) and 7 (period 128-256) owing to the common cosine function (frequency) between group I and III (i.e., $Y_{1}$ ), and group II and III (i.e., $Y_{2}$ ). Since at other timescales no significant common information is present, we do not see any linkage between the nodes as expected.

Case II. Here, we attempt to test the efficiency of the proposed approach for a non-stationary signal wherein the similarity between two different variables is changing with time. In this case, we generate three groups of time series following a similar approach as in the previous case except that the groups have a non-stationary relationship in terms of time. The mathematical formulation used for the generation of the time series (case II) is given in Table 1, and the common periodicities present between the three groups are shown in Figure 3 for easy visualization. Figure 3 shows that the common periodicity between all the 30 nodes is $S 1$ for the time period $t=1: 1000$ and $S 2$ for $t=1001: 2000$. Similarly, other details are intuitive from Figure 3. The particular cosine functions (signal) are plotted in Figures 4a-4c and the corresponding power spectra are shown in Figures $4 \mathrm{~d}-4 \mathrm{f}$.

For all 30 nodes presented, initially, we create a network using absolute correlation values of PCC (Fig. 5a) and then WMC (Figs. 5b-5h) by applying the 95 th percentile threshold. We observe that PCC only uncovers the internal connection among a group of nodes (Fig. 5a), whereas WMC captures the true relationship present in the signal of the 30 nodes (Figs. 5b-5h). For instance, in Figures 5c and 5 e all 30 nodes are significantly correlated corresponding to the presence of the signal $S 1$ and $S 2$ (Fig. 3) at scale 2 and 4, respectively. Similarly, nodes 11-30 have a common periodicity ( $S 3$, Fig. 3) at scale 7 hence the same is captured in Figure 5h. As expected, all other temporal scales do not show any significant correlation among the nodes.

Albeit, WMC is able to capture the dynamics of the non-stationary signal, still the time evolution pattern is not revealed completely. Hence, we propose to break the signal into two time windows $t=1: 1000$ and $t=1001$ : 2000, respectively. 

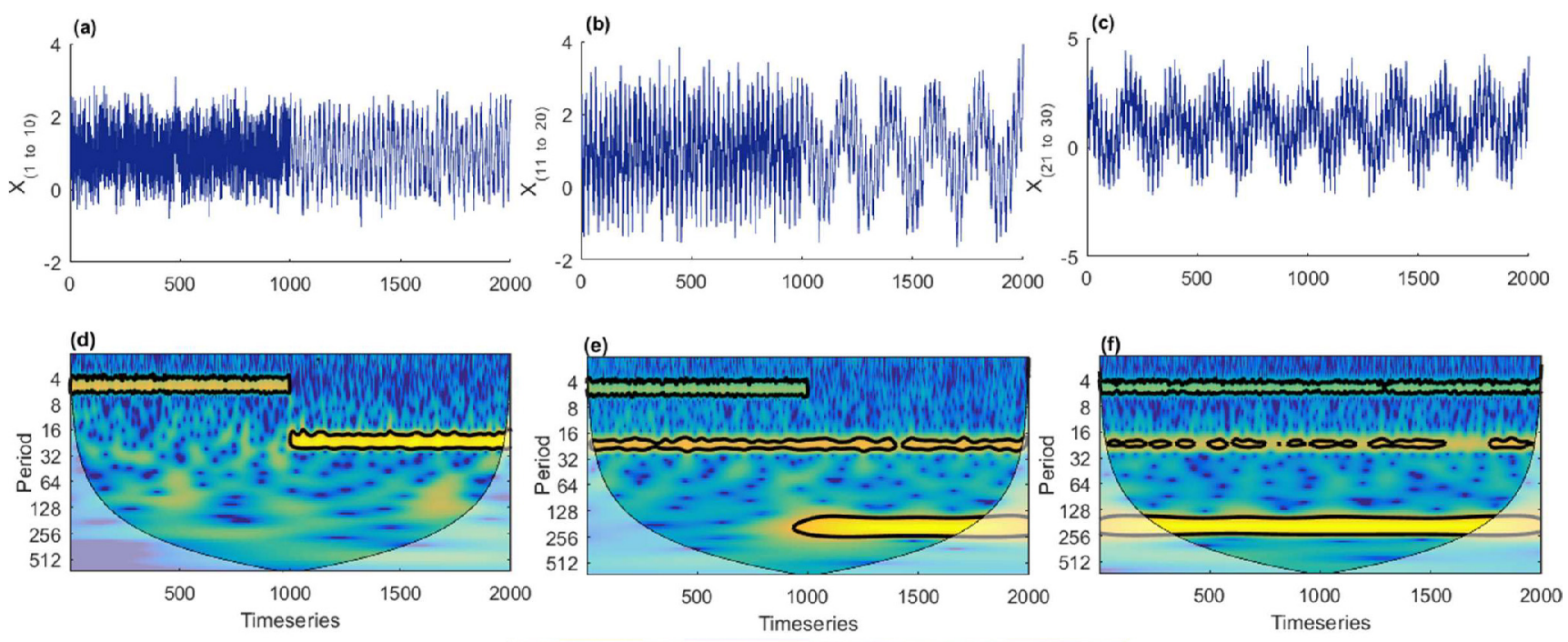

$\begin{array}{lllllllllllll}1 / 64 & 1 / 32 & 1 / 16 & 1 / 8 & 1 / 4 & 1 / 2 & 1 & 2 & 4 & 8 & 16 & 32 & 64\end{array}$

Fig. 4. The top row $(\mathrm{a}-\mathrm{c})$ shows the plot of the synthetic time series generated for network formation. Ten simulations were generated for each group of nodes. The bottom plot $(\mathrm{d}-\mathrm{f})$ shows the wavelet power spectra for one single time series from each of the group. The regions within the black contours denote the $95 \%$ significant values and the cones of influence denote the extent of the boundary distortion.

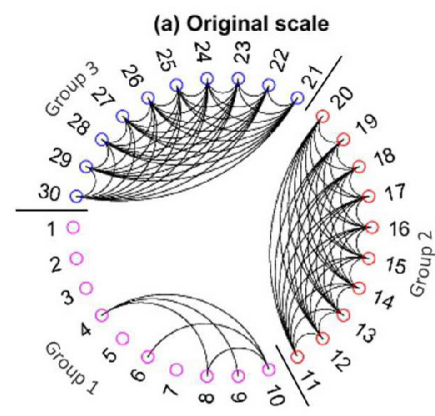

(e) Scale 4

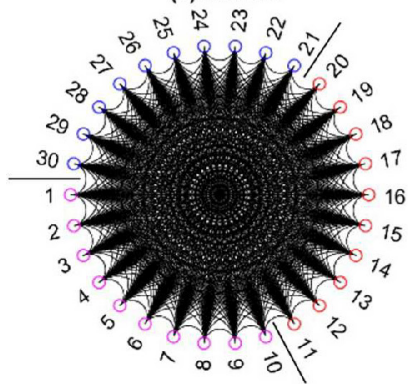

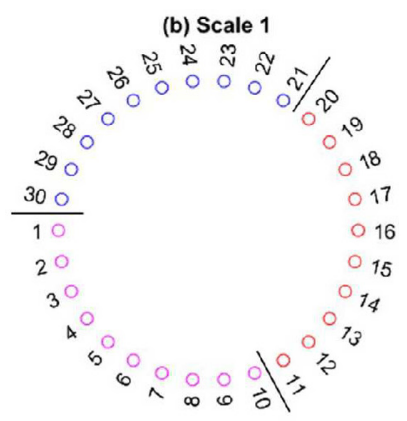

(f) Scale 5

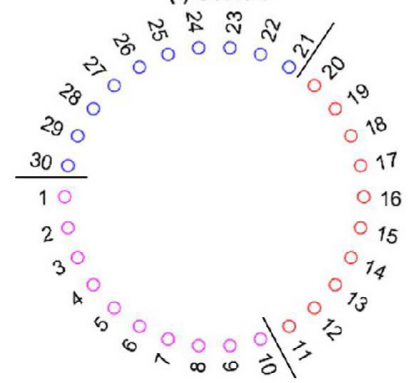

(c) Scale 2

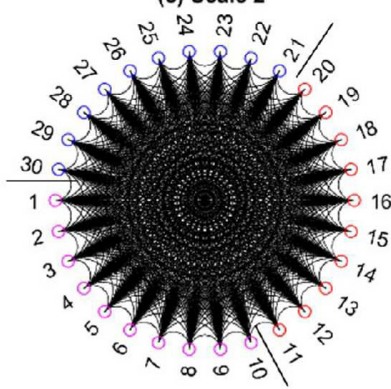

(g) Scale 6

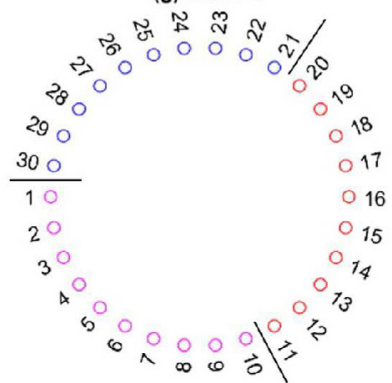

(d) Scale 3

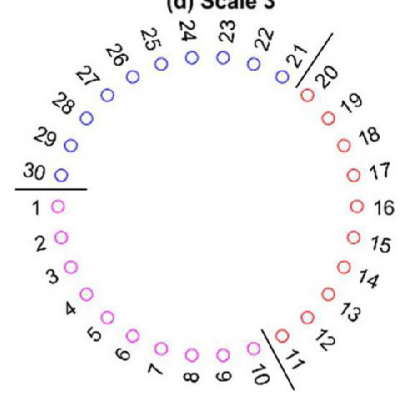

(h) Scale 7

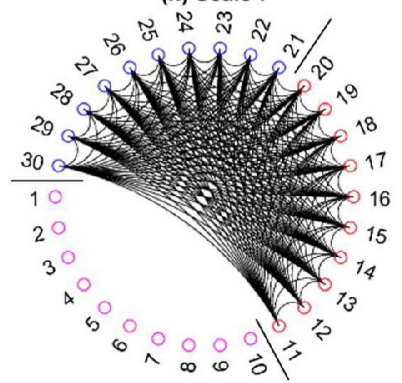

Fig. 5. Network structure and significant links obtained using the (a) PCC (original scale) and (b-h) WMC at scales $1-7$. A significant link is defined when the value of the correlation is higher than the 95th percentile.

For the time period $t=1: 1000$, the network formed using PCC (panel I, Fig. 6) does not show any links between the node groups even though there is a significant common periodicity between the nodes. However, constructing a network using WMC clearly captures the significant relationships between the node groups. For example, at scale 2 , all the node groups are connected with each other owing to the common periodicity $(S 1)$. Similarly, at scale 4, only nodes belonging to groups II and III are linked because of the common periodicity $(S 2)$. Further at scale 7 the significant links show the intra-group connections which are present in group III owing to the periodicity $(S 3)$.

Now, looking at the network formed for the time period $t=1001: 2000$, the PCC-based network (panel II, Fig. 6) does not clearly demarcate the changes in the signal, rather this network is very similar to what was observed during $t=1: 1000$ (panel I, Fig. 6). On the other hand, 
Panel I: $t=1: 1000$
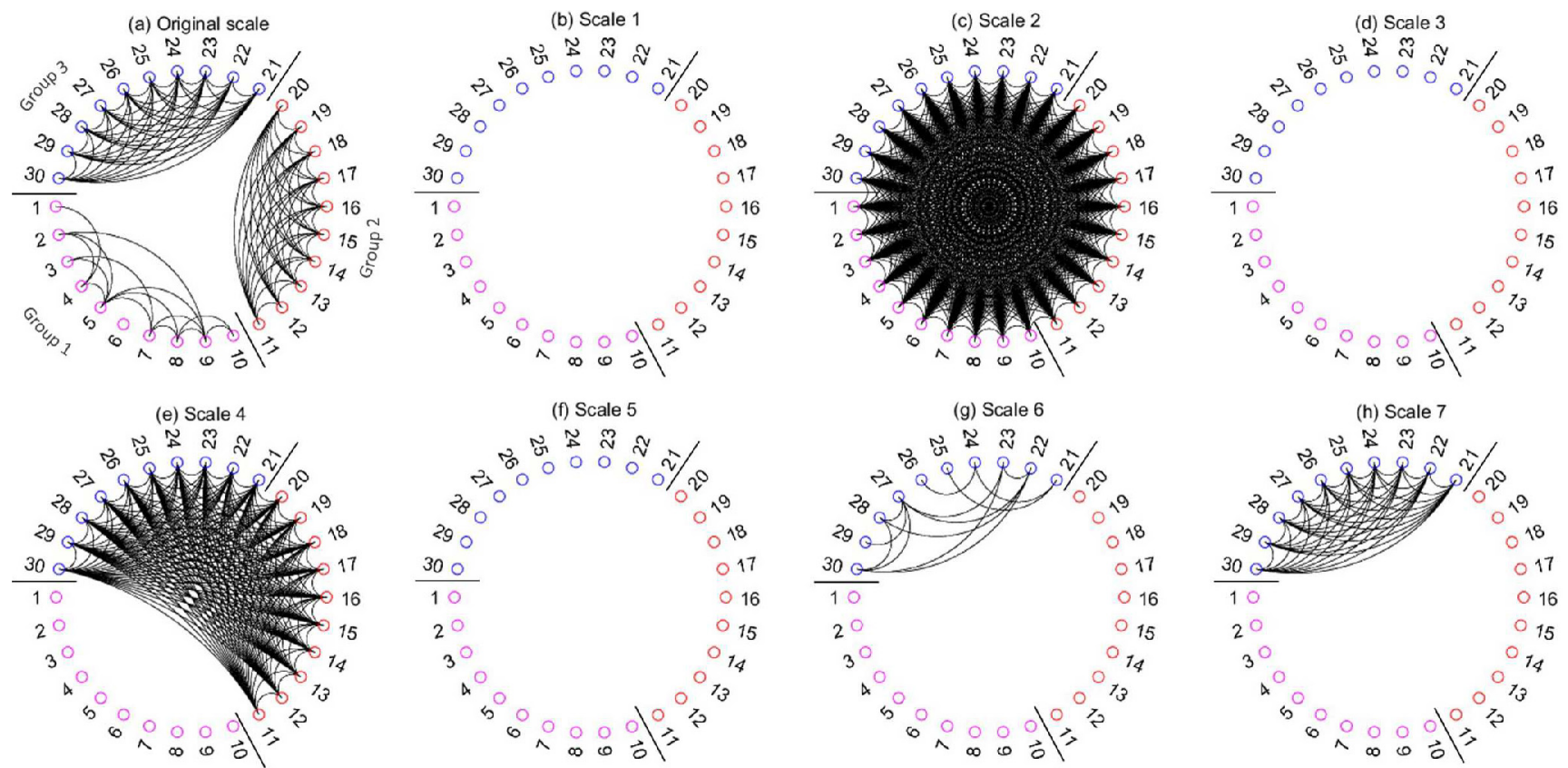

Panel II: $t=1001: 2000$
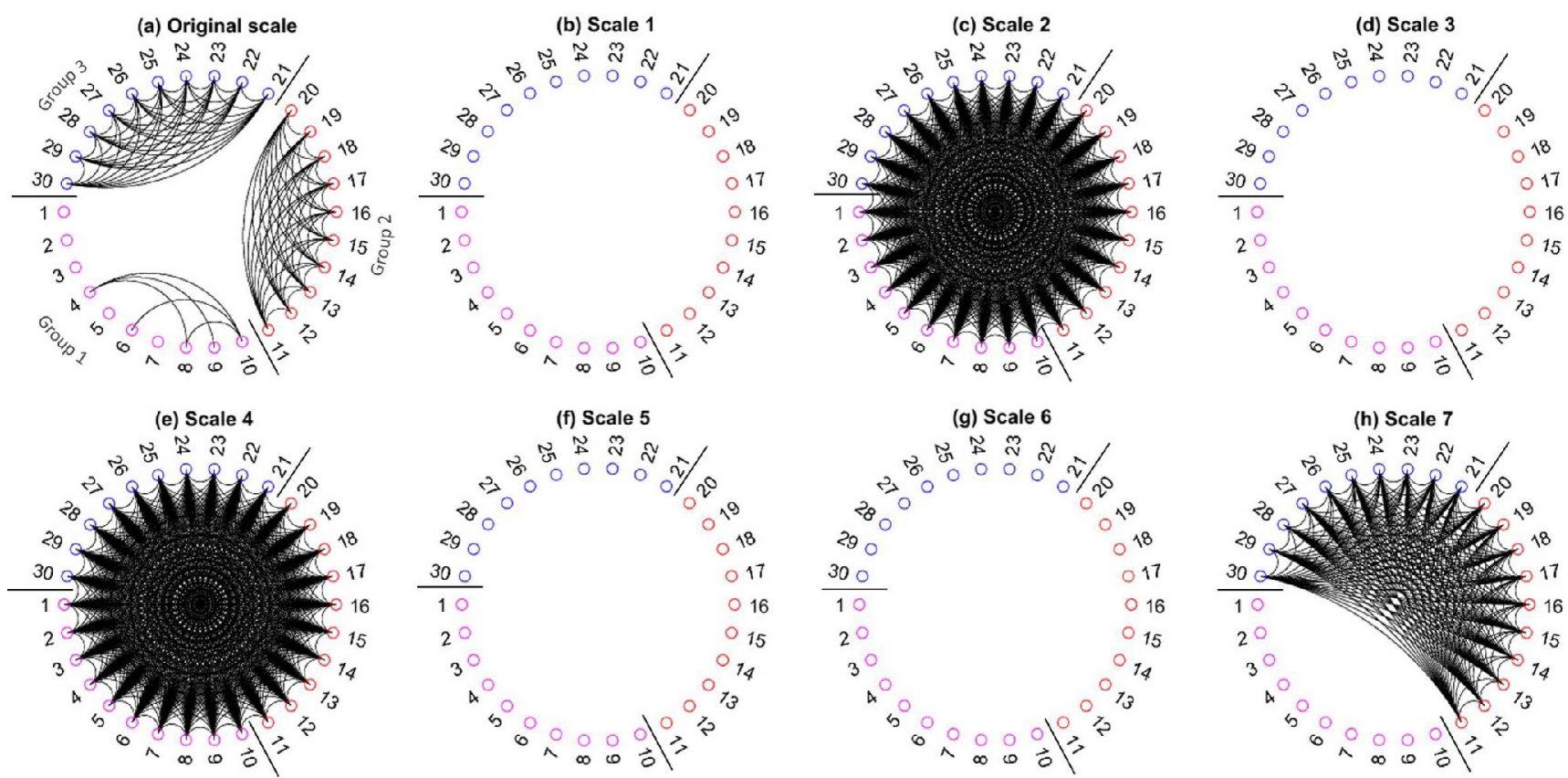

Fig. 6. Network structure and significant links obtained using the PCC (original scale) and WMC at scales 1-7. A significant link is defined when the value of correlation is higher than the 95 th percentile. Panel I corresponds to $t=1: 1000$ and panel II corresponds to $t=1001: 2000$. The few scattered links at scale 6 might be credited to the overlap/spill of information across scales in the wavelet transform.

the network formed using the WMC measure clearly delineates the changes in the connection between the node groups. For instance, the common periodicity between all the node groups in $S 2$ and that is clearly captured at scale 4 . Similarly, the significant links at scale 7 between the nodes belonging to group II and III are due to the presence of the common periodicity $(S 3)$. The intra-group links present between nodes $21-30$ at scale 2 is due to the presence of periodicity $S 1$ in that group of nodes. Furthermore, few scattered links at scale 6 might be credited to 
the overlap/spill of information across scales in the wavelet transform. This phenomenon is more dominant at higher scales since moving from a finer to a coarser scale the width between the scales decreases (for more details refer to Addison [38] and Polikar [51]).

Thus from this case study, we conclude that the WMC measure clearly captures the non-stationary relationship between the nodes and also clearly unravels at which scale the changes occurred.

From the presented two case studies, it can be inferred that the PCC is unable to capture the significant multiscale similarity between two variables, whereas the proposed WMC categorically captures the similarity at the specific time scales.

\section{Real world application}

To illustrate the applicability of the proposed multiscale similarity measure on real-world data, we present the global monthly SST network at different scales constructed using PCC and WMC measure. Here, we have used monthly gridded temperature data of $2^{\circ} \times 2^{\circ}$ grid resolution for the period 1979-2015. The data is provided by the NOAA/OAR/ESRL PSD and freely available at https://www.esrl.noaa.gov/psd/. As a preprocessing step of the data we have removed all grid points of SST with missing values or gaps, hence a total of 9456 grid points are considered in this study; and further, we calculate anomaly series by subtracting the mean for each month from each time series.

Initially, we construct the network using absolute values of PCC similarity measure on an observed scale, and then we begin the network construction at another temporal scale based on the WMC measure between each pair of nodes. By doing so, we generate a similarity matrix $\left|\rho^{l_{j}}\right|$ of size $9456 \times 9456$ on a particular scale $l_{j}$ and apply the $\theta=95$ th percentile threshold to obtain the adjacency matrix $(A)$. In this study, the maximum scale $J$ was taken as 7 owing to the distortion created due to the boundary effects of wavelet transform [52].

The comparison of the results obtained using PCC and WMC are shown in Figures $7 \mathrm{a}$ and $7 \mathrm{~b}-7 \mathrm{~h}$. We present the 95th percentile values of $\left|\rho^{l_{j}}\right|$ at each SST grid point to all other grid points, which is equivalent to the number of other nodes this node is highly correlated to (i.e., corresponds to the network measure degree). Additional plots (Figs. A.1 and A.2) that show positive and negative values of $\rho^{l_{j}}$ separately are presented in the Appendix.

Analysis of the PCC-based plot (Fig. 7a) shows that regions around the Indian and the Pacific Ocean are correlating on the order of $\approx 0.60$ as well as regions in the North Atlantic, the plot does not reveal on what time scales these correlations are the most prominent. It also does not reveal whether there exists one link between all regions of a high correlation or whether there are several different links between these regions. This emphasizes the need for a more sophisticated multiscale analysis measure like the WMC as analyzing the $\left|\rho^{l_{j}}\right|$ values at different scales results in far more detailed findings in the SST patterns as described in the following.
Moving from lower to higher temporal scales we find that there is not much significant interaction between SST at the finer, intra-annual temporal scales (up to 4-8 months). This can be expected as we are working with monthly anomalies and thus removed the annual cycle from the data. However, at the 8-16 months scale (Fig. 7e), we observe two zones with a relatively large number of significant connections in the equatorial Pacific and the Indian Ocean that are in accordance with the pattern observed during the ENSO phase and the Indian Ocean Dipole (IOD) which are known to impact each other via the atmosphere [53-55]. When looking at the signs of the correlation one can see that the ENSO tongue pattern west of South America is positively correlated with the SST pattern in the Indian Ocean (Fig. A.1e) and both are negatively correlated with the horseshoe pattern about the ENSO tongue (Fig. A.2e). This is the canonical SST pattern associated with an ENSO event and the corresponding IOD phase that can be excited by the ENSO event [24]. At inter-annual scale (16-32 months, Figs. 7f, A.1f, A.2f), these patterns become more pronounced as at least ENSO events can be predicted and act on scales of up to 2 years [56].

At the scale of $32-64$ months (Fig. $7 \mathrm{~g}$ ), the region of high correlation in the Eastern Pacific Ocean is fading away but coexist with a spot of high correlations on the Northern Pacific - forming together a pattern known as Pacific Decadal Oscillation (PDO) which has been in a warm phase since the late $1970 \mathrm{~s}$, but with time intervals of the cold phase, this variability is forced by Rossby waves [57] and can be seen in our analysis. Additionally, a new spot of high correlations around the subtropical part of the North Atlantic Ocean appears. The latter becomes more prominent on the 64-128 months scale (Fig. $7 \mathrm{~h}$ ). It resembles the SST patterns that are linked with the NAO [58] an atmospheric mode affecting SST. This becomes even clearer when analyzing the positive correlations (Fig. A.1h). One can see that the region in the subpolar North Atlantic and the subtropical North Atlantic are positively correlated, a known response of the ocean surface temperatures to the NAO. When focusing at the regions that have a high negative correlation with the other nodes, the most prominent feature is the correlation between the Southern Ocean around Antarctica and the subtropical/tropical the Pacific Ocean, west of South America. This correlation indicates what has just been confirmed by Ferster et al. [59]: a link between SST in the Southern Ocean and the Southern Annular Mode (SAM) with teleconnections to ENSO events [59]. As the SAM has a positive trend over the analyzed time period (1979-2015), this pattern emerges on the long time scales of several years. The positive states of SAM correlate with negative (cooling) trends in SST in the high-latitude Southern Ocean and positive (warming) trends within the Southern Hemisphere sub-tropics and mid-latitudes [59].

In conclusion, it can be said that the changes in the correlation patterns in the SST field when viewing at the different scale regimes as well as the difference in patterns between the positive and negative correlation strength show the complexity of the climate system and emphasize the importance of detecting correlations at multiple 

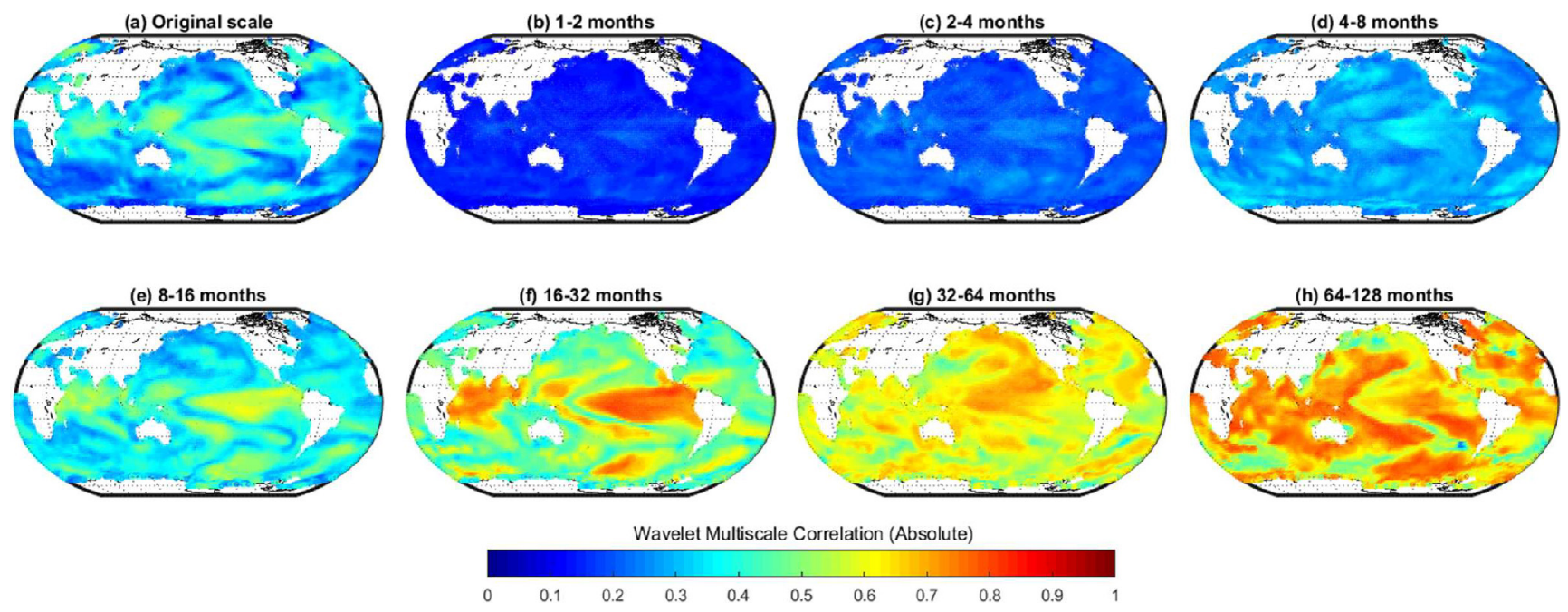

Fig. 7. 95th percentile of absolute wavelet multiscale correlation (WMC) values for the SST network constructed on multiscale using the PCC (original scale) and WMC at scales 1-7. Color represents the strength of correlation on each scale.

time scales. The PCC-based plot (Fig. 7a) on the original scale does not give the information needed to divide between the different modes of climate variability that are affecting SSTs in different regions and on different timescales. But by using the WMC measure instead of PCC, we can re-confirm known physics-based associations such as the well-known ENSO pattern in the Pacific at interannual scale (16-32 months, Fig. $7 f$ ), the NAO in the North Atlantic at intradecadal scale (Fig. 7g), but also the coupling of ENSO and IOD at interannual (Fig. 7f) and interdecadal (Fig. 7h) scales as well as the coupling between SAM, Southern Ocean SST and ENSO at the interdecadal scale (Fig. 7h). These findings are vital in (a) assigning the right temporal scales to the specific climate phenomena, (b) improving climate and ocean models. This implicitly affirms the validity of our approach and furthermore shows the efficacy of the method in detecting a variety of climate modes.

The proposed approach therefore clearly highlights the importance of advanced detecting methods, like the WMC, when dealing with such complex and big data, like global SSTs.

\section{Conclusion}

We have introduced a novel methodology to infer multiscale interactions from observations of dynamical climate systems that evolve over diverse temporal scales. The adopted metric - WMC - relies on the direct estimation of scale-by-scale correlations in the wavelet domain and is formulated here as an ensemble statistics across different temporal subsamples, with associated scale-dependent significance levels. Using a range of prototypical situations and a real-world case study, it is evident that the analysis at single observational scales using Pearson's correlation is not enough to capture the underlying multiscale features of complex systems with many nodes, as it is often the case with geophysical data, and thereby resulting in poor network reconstruction.

On the other hand, the significance of the results using WMC shows its ability to unravel the hidden similarity structure at different temporal scales. The obvious advantages of the proposed approach over the other single scale measures are (i) we can study the cross-scale relationship among dataset and systems on different time scales, (ii) network obtained using the WMC measure offer a wider perspective to unravel the network scale varying dynamics, and (iii) efficient in dealing with multiscale nonstationary complex systems, since, as shown, the effect of non-stationary can be handled with the help of wavelets. On investigating global SSTs at multiscale, i.e., with the WMC measure, new insights were obtained which had remained hidden at the observational scale, i.e., a simple PCC. Our findings re-confirm the known climate modes that affect SST like the ENSO patterns in the Pacific Ocean at inter-annual scale and the NAO that affects SST in the North Atlantic on the intra-annual scale. We also detect the coupling of ENSO and IOD at inter-annual and interdecadal scale as well as the coupling between SAM, Southern Ocean SST, and ENSO. We thereby affirm the validity of the WMC measure to detect climate modes on different temporal and spatial scales.

The proposed approach offers a new paradigm and possibilities for applications in other natural processes where interactions at multiple time scales exist e.g. in neuroscience, ecology, and economy. By studying on what time scale the factors are related and how the network properties emerge at that time scale, we may be in a better position to interpret the complex system and provide accurate predictions for future conditions. As a future work, the investigation could be extended to understand the causal effect of different climate drivers using the proposed WMC measure. This might give an understanding of the time scale relationships, which could be used to detect, attribute, and understand complex systems. 
This research for authors (AA, NM, and JK) was funded by Deutsche Forschungsgemeinschaft (DFG) (GRK 2043/1) within the graduate research training group Natural risk in a changing world (NatRiskChange) at the University of Potsdam (http://www.uni-potsdam.de/natriskchange). RM would like to thank the Humboldt Foundation for supporting this research through the Alexander Von Humboldt Fellowship award. Also, he would like to thank DST, India for the Inspire Faculty Fellowship Award. The authors gratefully thank Roopam Shukla (Department of Natural Resources, TERI University, New Delhi), and Niharika Tyagi (TERI University, New Delhi) for helpful suggestion and reading the paper.

\section{Author contribution statement}

AA and RM jointly conceived the presented idea. AA developed a theoretical framework, performed computations and validated methodology on synthetic case studies. AA downloaded and preprocessed SST dataset, prepared all figures and took lead to write the main text of the manuscript. LC helped to draw a meaningful conclusion from the real-world case study. All authors discussed the results, drew conclusions, and contributed to the manuscript text. RM, NM and JK supervised the project.

\section{References}

1. J.F. Donges, Y. Zou, N. Marwan, J. Kurths, Eur. Phys. J. Special Topics 174, 157 (2009)

2. A. Gozolchiani, K. Yamasaki, O. Gazit, S. Havlin, EPL (Europhys. Lett.) 83, 28005 (2008)

3. M.J. Halverson, S.W. Fleming, Hydrol. Earth Syst. Sci. 19, $3301(2015)$

4. A. Rheinwalt, B. Goswami, N. Boers, J. Heitzig, N. Marwan, R. Krishnan, J. Kurths, in Machine Learning and Data Mining Approaches to Climate Science, edited by V. Lakshmanan, E. Gilleland, A. McGovern, M. Tingley (Springer International Publishing, Cham, 2015), pp. 23-33

5. K. Steinhaeuser, A.R. Ganguly, N.V. Chawla, Clim. Dyn. 39, 889 (2012)

6. K. Yamasaki, A. Gozolchiani, S. Havlin, Phys. Rev. Lett. 100, $228501(2008)$

7. A.A. Tsonis, K. Swanson, S. Kravtsov, Geophys. Res. Lett. 34, L13705 (2007)

8. R. Quian Quiroga, T. Kreuz, P. Grassberger, Phys. Rev. E 66, 041904 (2002)

9. N. Malik, B. Bookhagen, N. Marwan, J. Kurths, Clim. Dyn. 39, 971 (2012)

10. U. Ozturk, D. Wendi, I. Crisologo, A. Riemer, A. Agarwal, K. Vogel, J.A. López-Tarazón, O. Korup, Sci. Total Environ. 626, 941 (2018)

11. T. Kreuz, M. Mulansky, N. Bozanic, J. Neurophysiol. 113, $3432(2015)$

12. A.J. Butte, I.S. Kohane, Mutual information relevance networks: functional genomic clustering using pairwise entropy measurements, in Biocomputing 2000 (World Scientific, 1999), pp. 418-429

13. J.I. Deza, M. Barreiro, C. Masoller, Chaos 25, 033105 (2015)
14. M. Paluš, in Advances in Nonlinear Geosciences, edited by A.A. Tsonis (Springer International Publishing, Cham, 2018), pp. 427-463

15. M. Rosvall, C.T. Bergstrom, Proc. Natl. Acad. Sci. 104, 7327 (2007)

16. A. Agarwal, N. Marwan, M. Rathinasamy, B. Merz, J. Kurths, Nonlinear Process. Geophys. 24, 599 (2017)

17. A. Agarwal, N. Marwan, R. Maheswaran, B. Merz, J. Kurths, J. Hydrol. 563, 802 (2018)

18. U. Ozturk, N. Marwan, O. Korup, H. Saito, A. Agarwal, M.J. Grossman, M. Zaiki, J. Kurths, Chaos 28, 075301 (2018)

19. A. Abbasi, L. Hossain, in Complex Networks, edited by R. Menezes, A. Evsukoff, M.C. González (Springer, Berlin, Heidelberg, 2013), pp. 1-7

20. P. Basaras, D. Katsaros, L. Tassiulas, Computer 46, 24 (2013)

21. N. Boers, A. Rheinwalt, B. Bookhagen, H.M.J. Barbosa, N. Marwan, J. Marengo, J. Kurths, Geophys. Res. Lett. 41, 7397 (2014)

22. N. Boers, R.V. Donner, B. Bookhagen, J. Kurths, Clim. Dyn. 45, 619 (2015)

23. N. Marwan, J.H. Feldhoff, R.V. Donner, J.F. Donges, J. Kurths, IEICE Proc. Ser. 1, 231 (2014)

24. K. Li, Z. Gao, X. Zhao, Physica A 387, 2981 (2008)

25. D. Looney, A. Hemakom, D.P. Mandic, Proc. R. Soc. A: Math. Phys. Eng. Sci. 471, 20140709 (2014)

26. A. Molini, G.G. Katul, A. Porporato, J. Geophys. Res. 115, 14123 (2010).

27. C.A. Varotsos, M.N. Efstathiou, A.P. Cracknell, Atmospheric Chem. Phys. 13, 5243 (2013)

28. J.A. Hatala, M. Detto, D.D. Baldocchi, Geophys. Res. Lett. 39, L06409 (2012)

29. C. Sturtevant, B.L. Ruddell, S.H. Knox, J. Verfaillie, J.H. Matthes, P.Y. Oikawa, D. Baldocchi, J. Geophys. Res.: Biogeosci. 121, 188 (2016)

30. E. Casagrande, B. Mueller, D.G. Miralles, D. Entekhabi, A. Molini, J. Geophys. Res.: Atmos. 120, 7555 (2015)

31. D.G. Miralles, A.J. Teuling, C.C. van Heerwaarden, J. Vilà-Guerau de Arellano, Nat. Geosci. 7, 345 (2014)

32. G.S. Okin, A.J. Parsons, J. Wainwright, J.E. Herrick, B.T. Bestelmeyer, D.C. Peters, E.L. Fredrickson, BioScience 59, 237 (2009)

33. D.P.C. Peters, B.T. Bestelmeyer, M.G. Turner, Ecosystems 10, 790 (2007)

34. J. Fernández-Macho, Physica A 391, 1097 (2012)

35. R.M. Lark, R. Webster, Eur. J. Soil Sci. 52, 547 (2001)

36. C. Yang, B. Olson, J. Si, Neural Comput. 23, 215 (2011)

37. S. Achard, J. Neurosci. 26, 63 (2006)

38. P.S. Addison, Physiol. Meas. 26, R155 (2005)

39. A. Agarwal, R. Maheswaran, V. Sehgal, R. Khosa, B. Sivakumar, C. Bernhofer, J. Hydrol. 538, 22 (2016)

40. H. Eryilmaz, D. Van De Ville, S. Schwartz, P. Vuilleumier, NeuroImage 54, 2481 (2011)

41. S. Kim, F. In, J. Empir. Financ. 12, 435 (2005)

42. V.N. Livina, N.R. Edwards, S. Goswami, T.M. Lenton, Q. J. R. Meteor. Soc. 134, 941 (2008)

43. S. Podtaev, M. Morozov, P. Frick, Cardiovasc. Eng. 8, 185 (2008)

44. M. Rathinasamy, R. Khosa, J. Adamowski, S. Ch, G. Partheepan, J. Anand, B. Narsimlu, Water Resour. Res. 50, $9721(2014)$ 
45. J. Richiardi, H. Eryilmaz, S. Schwartz, P. Vuilleumier, D. Van De Ville, NeuroImage 56, 616 (2011)

46. E. Shusterman, M. Feder, IEEE Trans. Image Process. 3, 207 (1994)

47. D.B. Percival, in Nonlinear Time Series Analysis in the Geosciences, edited by R.V. Donner, S.M. Barbosa (Springer, Berlin, Heidelberg, 2008), pp. 61-79

48. A. Agarwal, R. Maheswaran, J. Kurths, R. Khosa, Water Resour. Manag. 30, 4399 (2016)

49. K. Steinhaeuser, N.V. Chawla, A.R. Ganguly, Stat. Anal. Data Min. 4, 497 (2011)

50. W. Hu, B.C. Si, Hydrol. Earth Syst. Sci. 20, 3183 (2016)

51. R. Polikar, Fundamental concepts and an overview of the wavelet theory, The Wavelet Tutorial Part I, Rowan University, College of Engineering Web Servers 15, 1996

52. D.B. Percival, A.T. Walden, Wavelet Methods for Time Series Analysis (Cambridge University Press, Cambridge, 2000)
53. J.-J. Luo, R. Zhang, S.K. Behera, Y. Masumoto, F.-F. Jin, R. Lukas, T. Yamagata, J. Clim. 23, 726 (2010)

54. M.F. Stuecker, A. Timmermann, F.-F. Jin, Y. Chikamoto, W. Zhang, A.T. Wittenberg, E. Widiasih, S. Zhao, Geophys. Res. Lett. 44, 2481 (2017)

55. T. Yamagata, S.K. Behera, J.-J. Luo, S. Masson, M.R. Jury, S.A. Rao, in Geophysical Monograph Series, edited by C. Wang, S.P. Xie, J.A. Carton (American Geophysical Union, Washington, D.C., 2013), pp. 189-211

56. D. Chen, M.A. Cane, A. Kaplan, S.E. Zebiak, D. Huang, Nature 428, 733 (2004)

57. M. Newman, M.A. Alexander, T.R. Ault, K.M. Cobb, C. Deser, E. Di Lorenzo, N.J. Mantua, A.J. Miller, S. Minobe, H. Nakamura, N. Schneider, D.J. Vimont, A.S. Phillips, J.D. Scott, C.A. Smith, J. Clim. 29, 4399 (2016)

58. M.H. Visbeck, J.W. Hurrell, L. Polvani, H.M. Cullen, Proc. Natl. Acad. Sci. 98, 12876 (2001)

59. B. Ferster, B. Subrahmanyam, A. Macdonald, Remote Sens. 10, 331 (2018) 


\section{Appendix}
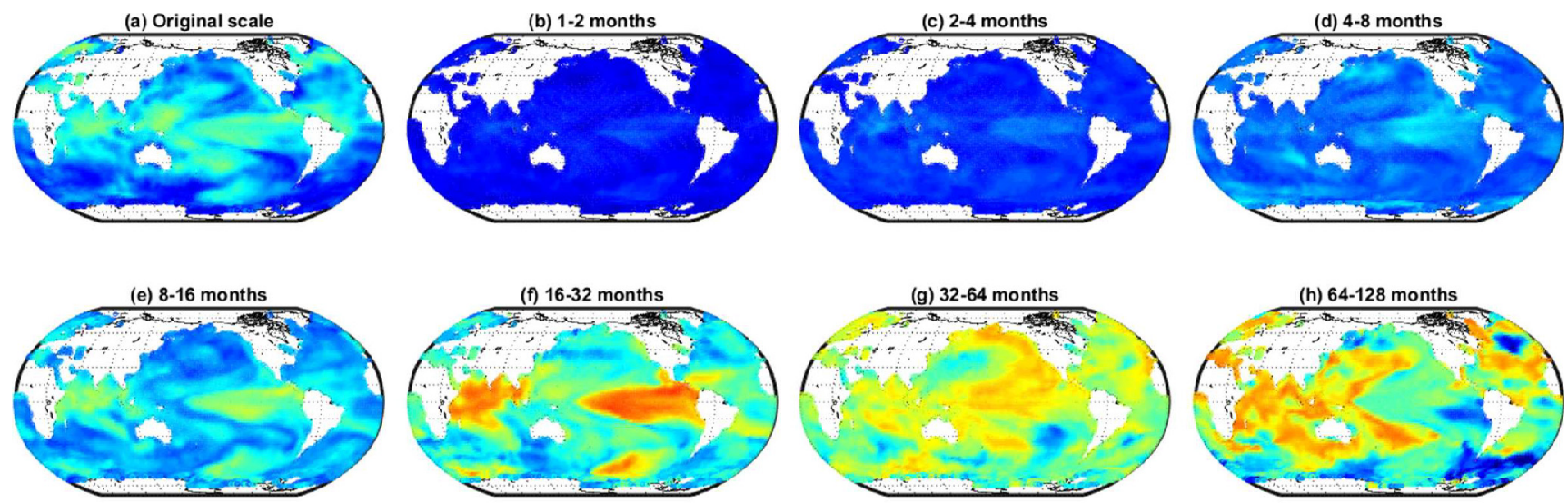

Wavelet Multiscale Correlation (Positive)

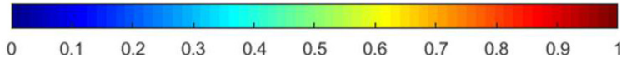

Fig. A.1. 95th percentile of positive wavelet multiscale correlation (WMC) values for the SST network constructed on multiscale using the PCC (original scale) and WMC at scales 1-7. Color represents the strength of correlation on each scale.
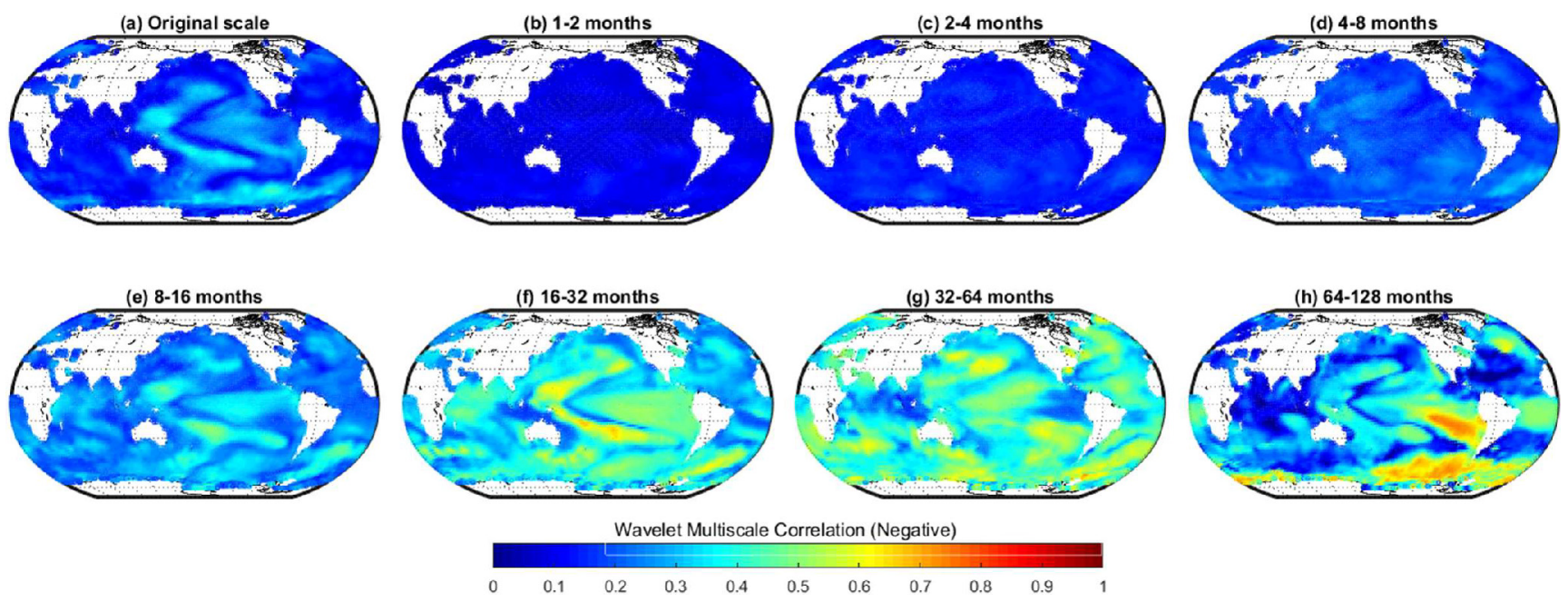

Fig. A.2. 95th percentile of negative wavelet multiscale correlation (WMC) values for the SST network constructed on multiscale using the PCC (original scale) and WMC at scales 1-7. Color represents the strength of correlation on each scale. 